
\title{
$\begin{array}{ll}\text { Research Square } & \begin{array}{l}\text { Preprints are preliminary reports that have not undergone peer review. } \\ \text { They should not be considered conclusive, used to inform clinical practice, } \\ \text { or referenced by the media as validated information. }\end{array}\end{array}$
}

\section{SUPPLY SIDE READINESS FOR UNIVERSAL HEALTH COVERAGE: ASSESSING SERVICE AVAILABILITY AND BARRIERS IN REMOTE AND FRAGILE SETTING.}

\author{
Veenapani Rajeev Verma ( $\nabla$ veenapani.verma1@gmail.com ) \\ Indian Institute of Technology Madras \\ Umakant Dash \\ Indian Institute of Technology Madras
}

\section{Research article}

Keywords: Service availability, Readiness, Supply side barriers, Difficult Setting

Posted Date: June 22nd, 2019

DOI: https://doi.org/10.21203/rs.2.10546/v1

License: (c) (1) This work is licensed under a Creative Commons Attribution 4.0 International License. Read Full License

Version of Record: A version of this preprint was published at Journal of Health Management on September 8th, 2021. See the published version at https://doi.org/10.1177/09720634211035211. 


\section{Abstract}

Background: The study hinged upon unravelling supply side readiness and barriers in attaining universal health coverage in a difficult setting. This district representative study is conducted in a fragile, remote, rural district of Jammu and Kashmir in India with unprecedented geographical barriers and heavy military deployment. Hilly geographical terrain, military skirmishes and sporadic militant attacks, rudimentary/absence of road network and absolute poverty are quintessential to this area. Methods: Mixed method approach was employed to triangulate quantitative and qualitative findings. Facility survey at various levels of facilities was conducted to gauge general service availability and service specific availability (depth of coverage). Compendium of checklist was designed using national standards in form of standard core questionnaire and parsimonious indices were computed by coalescing an array of tracer indicators across various domains as proposed in WHO's Service Availability and Readiness Assesment (SARA) module. Polychoric principal component analysis was used to identify significant variables causing variation in health service delivery and generalized ordinal logistic model was employed to determine factors impacting facility readiness score. Multifarious techniques like observations, key informant interviews and focus group discussions using semi structured questionnaires on both leaders and laggards were administered for critical stakeholder's analysis to discern qualitative information. Results: Results indicated poorest readiness for peripheral rural facilities with a composite score of $41 \%$ and $24 \%$ for subcenters and new type primary health centers respectively. Availability of basic amenities, diagnostic capacity and preparedness for emergencies and Non Communicable diseases was particularly subjacent having lowest scores. For primary care facilities; principal component was mainly characterized by basic newborn care as well as preparedness for delivery. Degree of environmental vulnerability of facilities, facility type and frequency of monitoring/supervision significantly impacted facility's readiness. Lack of incentives for health workers in remote and shelling prone areas, unavailability of residential accommodation, absence of motorable roads, political interferences aiding internal adjustments in form of transfer/attachment of health workers, leakages in supply chain of drugs and consumables, reticence of skilled staff in serving militancy impacted areas, nonchalant attitude of policymakers were identified as major barriers for service provisioning.

\section{Introduction:}

It has been unanimously acknowledged that strong health systems are paramount to achieve health system goals. Indicator 3.8.1 of Sustainable Development Goal (SDG) targets coverage of essential health services (defined as average coverage of essential services based on tracer interventions including reproductive, maternal, newborn and child health, infectious diseases, NCDs, service capacity and access among general and most disadvantaged population). Underpinning the need to strengthen fragile, resource-constrained health systems is the recognition that weak health systems impede attainment of global and national targets, and are insufficiently resilient to prepare for - and respond to - crises. Despite strong consensus on need to strengthen health systems there are inadequate methods to assess 
hordes of indices which can inform policy makers on priority areas for improvement (Wanzala et al, 2019). Albeit, current research does not adequately capture the complex, inter-connected relationship between health system building blocks and the setting in which they are situated (Sherr, 2017). Assessing quality of care requires that certain criteria and standards are identified in order to translate general dimensions of quality into something parsimonious that can be measured and interpreted.

Structural measures in the Donabedian's paradigm of structure, process and outcomes gauges the care attributes of healthcare delivery settings in the setting where care occurs (Donabedian, 1988). The assessment of structural quality of care divulges if the care provided under conditions are conducive or inimical to provision of care. These measures are symptomatic with system's readiness; comprehensive assessment of which is pertinent to evidence based policymaking and optimal resource allocation by transcribing identification of bottlenecks in service delivery. Data for measuring structural dimension of quality care including facility infrastructure, staffing and clinical training is extracted from health facility records and surveys. Previous literature delving into health systems performance over represent large health facilities, circumventing lower level peripheral facilities providing first contact of care. (Macarayan et al, 2018). Assessing facility readiness is paramount as it connotes the capacity of facilities to provide essential care for resilience to health challenges. As countries around the world agreed to Declaration of Astana, reaffirming their commitment to strengthen primary healthcare systems as an essential step towards achieving UHC; it is opportune to explore strategies for targeted action by tracking the progress towards UHC in different contexts.

In the context of health being state subject in India, it is incumbent upon state governments to implement policies to achieve provisioning of accessible and affordable healthcare. Thus, it is imperative for local governance to formulate framework to provide essential basic package to its citizens and have critical discourses and conjectures on benefit package in regional context that can be transmuted into increased access at community level. Also, in the context of decentralization, estimation of standardized, replicable and comparable supply side readiness metric at subnational and disaggregated level is imperative for context specific evidence for prioritization of interventions but remains a colossal challenge due to lack of dependable and representative data sources. The systematic data and studies underscoring the levels, determinants and barriers to service availability at subnational level are rather limited for comprehensive and detailed assessment. Moreover, existing studies on service readiness are not exhaustive and doesn't circumscribe entire spectrum of services rather focuses on narrow unidimensional metric measured either by type of condition or type of intervention (Boerma, 2014; Islam et al, 2016; Ssempiira et al, 2018; Biswas et al,2018). Our study strives to address this gap by conducting comprehensive study and providing synoptic view by leveraging on small area methodologies enunciating the nuances of supply side readiness.

Health care access in difficult settings like rural, remote, and fragile zones poses unprecedented challenges prompting providers to confront myriad complexities stemming from paradoxical nature of setting in which they operate. Providers in contested borderlands has to reckon with peculiar threats generating a situation of unpredictability and uncertainty. They have to face number of hazards including 
stray bullets and shells coming from across border/mine related threats when operating at zero line. However, the border context of conflict is often neglected by researchers whereas it is germane to explore the multifaceted nature of conflict. This issue of insecurity impinging on functioning of health systems gets exacerbated by unfavorable conditions such as weak governance, geographical remoteness, reluctance of health workers to function in remote and fragile areas, harsh terrain and weather conditions. However, no attempt in existing studies has been made to incorporate these vulnerabilities from supply side dimension. However, our work attempted to succinctly summarize these vulnerabilities in a conflicted borderland and explore the impact of level of these vulnerabilities on the capacity of system to deliver care.

\section{Objectives:}

Objective of the study is to a) Evaluate the service readiness of health facilities and create a concise index subsuming plethora of discrete indicators and b) Ascertain supply side barriers in service provisioning via stakeholder's analysis. Study also strives to expand analytical domain unravelling context and area specific intricacies associated with service delivery

\section{Methods:}

\subsection{Area setting}

The study was conducted in Poonch district which is a high focus district of Jammu and Kashmir state in India. It is one of the remotest districts of Jammu and Kashmir with heavy military deployment as is bounded by Line of Control (porous boundary between Indian and Pakistani administered Kashmir and is one of the most complex frontier systems in the world) and is bearing the brunt of cease fire violations. The topography of district is hilly and mountainous barring few low lying valleys and Pir Panjal range separating it from Kashmir region. The district is also low performing in terms of health indicators and has been categorized as 'high priority district' by Ministry of Health and Family Welfare based on poor performance in health composite index. For decades the intricate linkage between the external dimension (Indo-Pak conflict) and internal dimension (militancy in the area) leading to proxy wars and military skirmishes exacerbated the ordeal of border population having an abode in this area. Multitude of security disruptions through shelling and firing and fear perpetuated by armed conflict is causing obstruction of access to health workers and patients, rendering the health system pregnable.

The district is divided into three medical blocks and all of them have been chosen for study. There are insurmountable healthcare access barriers in region which is reflective in suboptimal health facility coverage. Health facilities represented in survey were categorized as: a) Second tier District hospital and Community health centers( $\mathrm{DH}$ and $\mathrm{CHCs}$ ) providing secondary healthcare b) First tier Primary health centers(PHCs) serving as first point of contact between population and healthcare providers and c) Sub centers which are the peripheral health institution available to rural population. These components were assessed separately due to varying standards thus, disparate number of variables for various hierarchies 
of facilities were used as tracer indicators. As a fallout of armed militancy and infiltration of militants via porous borders, whole border is fenced and at numerous places fencing is done much inside zero line enclosing vast swathes of population and these lands are fenced and gated ensuing obstructions to access. Some of the health facilities and catchment population are inside this zero line and all of them have been included in the survey.

\subsection{Study Framework}

The study employed concurrent triangulation mixed method approach to corroborate quantitative findings with qualitative nuances where each method was implemented simultaneously and interactively. Triangulation is achieved by intentionally using more than one method of gathering and analyzing data about same phenomenon in order to seek convergence and corroboration and to eliminate inherent biases from using only one method (Greene et al, 1989). This methodology highlights the paradox, contradictions and new perspectives as any discordance between findings discerned from these two methods has huge policy implications. The representation of study framework is given in Fig 1.

Facility survey encompassing 90 Subcenters, 44 Primary health centers, 3 Community health centers and 1 District hospital was conducted to gauge general service availability and service specific availability (depth of coverage). Subcenters were stratified on the basis of location and degree of geographic remoteness. Data collection ensured district representativeness and was collected from all three medical zones in district. The survey elicited information on measures pertaining to performance and quality of health service delivery system. Compendium of checklist was designed using Indian Public Health Standards (IPHS) in form of standard core questionnaire and scorecard was generated for each facility.

Qualitative methods entailing use of multifarious techniques like observations, casual conversations, key informant interviews and focus group discussions using semi structured discussion guide on both leaders and laggards were administered for critical stakeholder's analysis. Participants and sample selection ensured saturation of data and potential transferability of findings to other contexts and settings. All transcripts from in depth interviews and discussions were recorded, transcribed verbatim and translated from local dialect to English. Qualitative data was analyzed thematically to explore emergent themes or divergent cases by manifest content analysis. Data was coded line by line and higher level themes were generated using NVivo 10 software. To triangulate the comparison of findings from disparate data sources, both quantitative and qualitative findings were mapped around dimensions of SARA.

\subsection{Data Collection Tool}

\subsubsection{Service Availability}


Service availability captures the physical presence of delivery of services encapsulating the domains of infrastructure, human resources for health and utilization. This doesn't capture the nuances and complex dimensions such as geographical barriers, travel time, condition of the infrastructure, quality and mix of health workforce, barriers faced by health workers and provider behavior as it requires more complex input data. The indicators are expressed as a percentage score, compared with benchmark set by WHO. Stakeholder's analysis explored the themes which were not in precinct of SARA methodology.

\subsubsection{General Service Readiness}

This paper focuses primarily on service delivery inputs, in the terminology of Service Delivery Indicators model, which is analogous to service readiness in the WHO SARA framework. Information was collected across dimensions of amenities, equipment, medicines, laboratory and infection control protocols as proposed in WHO's Service Availability and Readiness Assessment (SARA) methodology. The indicators were selected in tandem with national standards and guidelines of health system functioning encapsulating the system's readiness to deliver healthcare services and were categorized within SARA domain. All items were binary, with 1 indicating that item was observed present (and functional as applicable) and 0 indicating the item was not present or couldn't be assessed e.g. due to relevant service not being offered.

\subsubsection{Service Specific Readiness}

Refers to ability of health facilities to offer a specific service and the capacity to provide service measured through consideration of tracer items that include trained staff, guidelines, equipment, diagnostic capacity and medicines and commodities (WHO, 2013). The readiness of services embedded in essential/basic health package was assessed separately for 19 interventions and were chosen by triangulating national guidelines as envisaged in High Level Expert Group on Universal Health Coverage (Planning Commission of India, 2011), SARA guidelines (WHO, 2013) and comprehensive health care package outlined by Task Force on the roll out of Comprehensive Primary Health care, Ministry of Health and Family Welfare (Government of India, 2014). These package of services are symptomatic to quality assurance tool designed to ensure that necessary services are offered in accordance with standard protocol. The readiness scores were calculated for following set of service package-i) Routine Immunization ii) Family planning and Contraception iii) Maternal and child services iv) School health services and adolescent health v) Basic surgical care vi) Obstetric care vii) Outreach services viii) Malaria and febrile diseases ix) Disease control programs $\mathrm{x}$ ) General and Oral health xi) Tuberculosis and Leprosy services xii) HIV/STI diagnose and management xiii) Respiratory/Chest disease xiv) Cardiovascular diseases xv) Diabetes xvi) Cancer xvii) Mental health xviii) Neurology xix) Emergency preparedness.

\subsection{Data Analysis Tool}


The analysis was conducted separately for different tier of facilities. Service availability index subsuming three components a) Health services Infrastructure Index-Average score of three indicators: facility density, inpatient beds, and maternity beds. b) Health workforce index-Core health workers c) Service utilization index- Average score of two indicators: outpatient visits and hospital discharges. Further, General service readiness is derived by computing mean availability of tracer items for each subdomain by dividing the total number of facilities having tracer item available (value=1) by total number of facilities, multiplied by 100 to get a percentage value. An overall general readiness score was calculated based on unweighted mean of domains. Finally, Service specific readiness was gauged by calculating summary scores for each service based on proportion of availability of each tracer item.

Further, quantitative methods included Polychoric principal component analysis (PCA) for exploratory data analysis aiding feature identification. With PCA, reduced set of variables are generated that describes the commonality of a set of related indicators. The goal of PCA is to find components $z=\left[z_{1}, z_{2}\right.$, $\left.\ldots, z_{p}\right]$,which are a linear combination $u=\left[u_{1}, u_{2}, \ldots, u_{p}\right]$ of the original variables $x=\left[x_{1}, x_{2}, \ldots . . x_{p}\right]$ that achieve maximum variance. The first component $z_{1}$ is given by linear combination of original variables $x$ and accounts for maximum possible variance.

$Z_{i 1}=\varphi_{11} X_{1}+\varphi_{21} X_{2}+\ldots \ldots \ldots \ldots \ldots \ldots . . . . . \varphi_{p 1} X_{p}=\sum^{p}{ }_{j=1} \phi_{i 1} X_{i}(1)$

The second component captures most information not captured by first component and is also uncorrelated with first component. The proportion of variance in each original variable $x_{i}$ accounted for by first $c$ factors is given by sum of squared factor loading; i.e. $\sum_{c}{ }_{c} f^{2}{ }_{i k}$. When $c=p$ (all components are retained), $\sum^{k}{ }_{\mathrm{c}}{ }^{2}{ }_{\mathrm{ik}}=1$ (all variation in data are explained). The results were analyzed by variance distribution according to PCA factor loadings by approximating principal components with input variables demonstrating importance of variables based on proportion of variances contributed/explained by input variables. Factor loadings represented as $\varphi_{11}, \ldots . . . \varphi_{p 1}$ in equation 1 are the correlations between original variables $x$ and components/factors $z$, denoted as $F=\operatorname{cor}(x, z)=U D^{1 / 2}$. The components were rotated using oblique rotation in order to obtain sharper conceptual solutions to interpret prominent variables.

Since, PCA is only suitable for continuous data as it is developed for the samples from multivariate normal distribution, and most of the theoretical results, including the implicitly used consistency of the estimates of factor loadings, were derived under the normality assumption. In order to rectify this problem, a more suited, PCA using Polychoric correlation matrix was employed. Polychoric correlation is the correlation between two ordinal variables obtained as the maximum likelihood estimate under the assumption that the ordinal variables are obtained by coarsening a bivariate normal distribution (Kolenikov, 2016).

The method of Parallel Analysis (Horn, 1965) based on Monte Carlo extensions (Glorfeld, 1995) was implemented to make decisions about component retention. A critical aspect of Principal Component 
Analysis is the decision on number of factors to be retained which is important because varied methods are more or less likely to overestimate or underestimate number of components. Horn reasoned in a finite sample of size $\mathrm{N}$, is expected to see eigenvalues greater than and less than 1 simply because of 'Sample Bias'. Parallel analysis corrects this bias in Kaiser rule by generating sufficiently large number, $\mathrm{K}$ of uncorrelated random data of same dimension as observed data and thereby; performing parallel PCA and averaging the results. A strong consensus has developed in the literature endorsing parallel analysis as among the most accurate method for decision rule. Those components whose adjusted eigenvalues were greater than 1 were retained.

For ordinal multi-level categories of outcome variable, ordinal logistic regression (parallel-lines model/proportional odds model) would be parsimonious, intuitive and easy to interpret for modelling the impact of covariates on health facility readiness score. However, the assumptions embedded in parallellines model/proportional odds model are overly restrictive and often violated as it's probable for one or more $\beta$ 's to vary across $\mathrm{j}$. The constraints in ordered logit model is discerned by conducting Brant test. This test examines the null hypothesis that all estimated coefficients in ordered logit model satisfies the parallel odds assumption by conducting both global test as well as separate test for all independent variables. Indication of violation of proportional odds assumption insinuates ordered logit model is misspecified and alternate variant of ordered logit is adopted. In tandem with the results, partial proportional odds variant of generalized ordinal logit model was computed to unravel the determinants of health facility readiness. In our study, Brant test revealed that vulnerability and staff positions do not meet proportional odds assumption and their estimated coefficients vary across severity thresholds. Hence, partial proportional odds model which is a special case of generalized ordered model is fitted to overcome the lacunae inherent in parallel-lines model. It is a less restrictive model which allows the effect of independent variables to vary with the point at which categories of dependent variables are dichotomized. Partial proportional odds model is commonly denoted as:

[Due to technical limitations this formula could not be inserted here. It can be found in the formulas document in the supplemental files. This is formula (2)]

Where $X_{i}$ is a vector of explanatory variables, $\beta_{j}$ is a vector of estimable parameters and $a_{j}$ and $a_{(j-1)}$ are the upper and lower thresholds for readiness scores. $Y$ represents ordinal dependent variable and $M$ is the number of categories of ordinal dependent variable. A three category dependent variable was constructed i.e. Poor readiness, Fair readiness and Good readiness. In order to facilitate the interpretation and compute the direction and magnitude of determinants on facility readiness score, elasticities (marginal effects) were computed.

\section{Results}

\subsection{Service Availability}


Fig 2.1 summarizes the components of service availability in the district .There were 3.7 facilities (at all levels of hierarchy) per 10000 population as against the required norm of 2 per 10000 population culminating into a score of 1.6 for facility density. Inpatient bed density; a standardized indicator measuring levels of access to hospital inpatient services by designated populations was nondescript in our study area with only 7.13 beds per 10000 population yielding a score of $28 \%$. On the other end of spectrum, the score of maternal bed density was rather propitious at $85 \%$ indicating priority setting of Maternal and Child health programs. The health worker density is the health workforce indicator that is most commonly reported internationally and represents critical starting point for understanding health system resources situation in an area. The density of core health workers was 15.01 per 10000 health workers vis. a vis. the health workforce density threshold of 23. Nonetheless, the goal of universal health coverage requires a paradigm shift, going beyond a discourse on shortages but rather focusing more explicitly on accessibility, acceptability, quality and productivity of health workforce (WHO, 2014). Delving further into the workforce mix, it was revealed that the shortage of specialists and doctors was particularly striking with district having $54 \%$ vacancy of specialists and $76.6 \%$ vacancy of medical officers. There was only 1 doctor/6241 population as against the guideline of 1 doctor/1000 population. Doctor patient ratio in $\mathrm{DH}$ was $1 / 9880$ which is 10 times less than recommended ratio, $\mathrm{PHC}$ had a ratio of $1 / 5878$ with majority of doctors from Indian System of Medicines and no doctor was in position in any of the NTPHC. Strengthening service delivery and augmenting utilization are indispensable to improve health status and outcomes. Outpatient utilization that measures number of outpatient visits to health facilities during one year relative to the total population of same geographical area was 1.64 as compared to the target of 5 generating a score of $32.5 \%$. Inpatient utilization (hospital discharges per 100 population excluding deliveries) was $88.3 \%$ of target with 8.83 people getting hospitalized per 100 population.

Stakeholder's analysis explored these themes in detail that were not in precinct of SARA methodology. On the infrastructure front, even though results indicated favorable facility density scores; the condition of building was disconcertingly dilapidated and deficient posing challenges for effective service delivery. Subcenters, particularly were in decrepit condition and $74 \%$ were operating from rented premises.

"The entrance to facility isn't conducive for patients. There's scree slope and loose stones. Once an elderly man was maneuvering his way to facility; he slipped and broke his backbone and had to be admitted to district hospital and later referred to capital. I was consumed with guilt. But it's not in my hands, you see."

-Nursing Orderly, Subcenter

"It is becoming increasingly daunting to operate from this single, kutcha rented room with no storage facility. We are grappling with problem of thefts every year. There's no compound wall and there are gaps in window panes due to which locals break and steal furniture, medicines and even record registers. Entire untied funds gets dissipated in replacing lost items."

- Pharmacist, Subcenter 
In conjunction with shortage of specialists and physicians; absorption and retention of other workers was also identified as a colossal challenge in far flung and fragile area. Workers were are not willing to serve in our area setting due to confluence of dampening factors like isolation, difficult terrain, dense forests, absence of road and transportation network, shelling hazards, inaccessibility to housing and market and contractual mode of employment.

"Post rainy season jungles metamorphosis into dangerous form and there's burgeoning of grass and tall maize crops, making it a lurking ground for wild bears and providing recesses for militants. My frequency of visiting center plummets significantly during that season. Last season my ASHA worker was mauled by bear while going for vaccination outreach. My family is urging me to quit the job. I haven't been regularized for last 10 years and earning peanuts. Is it worth it?"

-Multipurpose worker, NTPHC

Due to non-existence of incentive structure, workers resort to liaison with local political leaders for internal adjustment to get themselves attached to district/block headquarters from their original location of posting; leaving remote area underserved. In the absence of such arrangements, when workers serve in remote areas, they resort to absenteeism and dereliction of duty. This causes challenges with distribution and skill mix of health workforce with more than sanctioned workers in easy to access areas and scarce and unmotivated workers in villages.

"I am a sole worker here; it feels like walking at knife edge trail. I start by 7am from home but can only reach facility by 12 noon after hiking for 2 hrs. I am forced to lock the facility by 2:00 pm for the fear of missing last vehicle back home. Administration is pestering me to stay near the facility; but where's the housing?"

-Multipurpose worker, Subcenter

Low utilization rates of ambulatory care are inextricably related to obstructed service delivery. However, in context of hilly and remote areas, high facility density score should be interpreted with caution as it doesn't necessarily translate to physical accessibility for dispersed population living in higher reaches. Some pockets are completely inaccessible warranting new facility/upgradation of lower tier facility manned with skilled worker.

"We have population of 14,000 in the village; for this population, a PHC is required especially to cater delivery services. From these far flung areas, we have to take our women to district headquarter for delivery after paying hefty amount to hire private vehicle as there's no public transport post $3 \mathrm{pm}$. Even for other ailments, we don't want to walk for hours only to find the facility closed or without drugs."

Sarpanch, Village Panchayat

\subsection{General Service readiness}


Hospitals had high readiness scores on an average (76\%) as compared to the new type primary health centers (24\%). The average item availability for basic equipment ranged from $29 \%$ to $89 \%$ with lowest being for new type primary health centers and highest for district hospital. The average score for standard precaution against infection was $60.2 \%$ across all level of facilities. The highest average score was noted in community health centers with a value of $79 \%$. Laboratory diagnostic capacity was subjacent for new type Primary health centers at meagre $4 \%$. The assessed items most likely to be unavailable even in high performing facilities were essential medicines and amenities.

PCA for subcenters and primary health centers revealed that two components extracted from 71 variables for subcenters explained $22 \%$ variance, whereas first two extracted components from 221 variables for primary health centers explained $31.59 \%$ variance. For each component, relative size of coefficients depicting the commonality in coefficients is illustrated in Fig 4. The principal component for subcenters was weighted most heavily on equipments whereas, second component was representative of medicines. Estimated coefficients on principal component for primary health centers on the other hand, had maximal relative strength on diagnostics and second component analogous to subcenters represented medicines.

\subsubsection{Amenities}

Around $6.66 \%$ sub centers reported having none of the tracer items symptomatic with amenities and one fourth facilities had less than $25 \%$ of 7 tracer items. However, only half of the sub centers had readiness score greater than $50 \%$. A tenuous $10 \%$ primary health centers were equipped with half of the 58 tracer items. None of the primary health center had communication network like email or NIC terminal although all of them were endowed with electricity having power backup and government ownership of building possessing adequate premises. However, only three PHC's had quarter for staff members and $40 \%$ didn't have residential arrangements for medical doctors. However, this was more pronounced for new type primary health centers where none of the facility had quarter for staff members and $88.88 \%$ were bereft of quarters for medical officers. Moreover, the electricity/power supply in new type primary health centers was intermittent as well with just two facilities having continuous power supply. Although, these facilities are characterized by geographical inaccessibility from settlements, and were marred by unavailability of ambulances and basic communication network. Hospitals i.e. district hospital and community health centers were found to be relatively accoutered with amenities Vis a Vis lower level of facilities.

Fig 5.1 and 5.2 illustrates the scree plots generated from PCA. Results of Horn's parallel analysis revealed one component having eigenvalue greater than 1 explaining $44.54 \%$ of common variance for sub center. The principal component was weighted heaviest for emergency transport and communication network. Concomitantly, first four components explained $65.5 \%$ common variance for PHC's and the principal component was characterized most strongly for infrastructural impediments like provision of quarters for medical officers and government ownership of building.

The stakeholder's narratives were consonant with the above findings and acknowledged incommensurable staff working conditions and nonresponsive emergency transport rendering staff 
members to derelict from duty and patients to incarcerate/die in transit as a barrier to service provisioning. Retaining health staff, particularly in remote and conflict areas is an insurmountable challenge and amenities play a crucial role in health staff decisions about absorbing and retaining posts. However, health workers bemoaned about dearth of personal accommodation both at health post and in surrounding community.

"Construction of quarters for medical officers started ten years back. It's still incomplete. Ever since I joined the facility, I have been sleeping in labour room with shell sprinters puncturing holes in the wall incessantly. When shell hits at/near the labour room, I shift to the general ward to sleep and vice versa."

-Medical officer (PHC at Zero Line)

Budget constraints was identified to be an Achilles heel stymying the funding of stalled projects and paying for the amenities.

"There is a serious concern pertaining to funds. We couldn't complete stalled projects, purchase needed ambulances or any bullet proof ambulance. We don't even have adequate funds to pay the monthly rent of facilities. There are no NGO's, no AID agencies and no PPP. We can't even provide incentives to attract workers in most disturbed areas"

-Senior district administrator

\subsubsection{Equipments}

There was perceptible variation in the availability of equipment in subcenters and hospitals. In subcenters, the availability of equipment ranged from $4 \%$ to $70 \%$ with the average availability of $40 \%$ and 12 facilities having less than $25 \%$ items. Striking difference in the readiness scores was found between PHC's and NTPHC's. None of the PHC had inventory of eye care equipment and only three PHC's were equipped with phototherapy unit and MVA/MTP suction aspirators whereas this list was protracted to enfold all the equipments in newborn baby corner and neonatal pediatric unit viz radiant warmer, laryngoscope/endotracheal equipment, mucus extractor and baby bassinet etc for NTPHC's. With the average equipment score of $29 \%$, NTPHC's score was abominable specifically for delivery preparedness and cold storage. The highest performing NTPHC merely possessed half of the tracer equipments on the day of survey. Community health centers and district hospital were devoid of the equipments pertaining to NCD care and none of the facility had e-ventilator for operation theatre, noninvasive ventilator, spirometer, dialysis machine, memography, stadiometer and colposcope etc. However, all the hospitals had basic equipments required for labour room and newborn corner. CHCs with a readiness score of $68 \%$ and district hospital with a score of $88 \%$ were efficacious vis a vis primary care facilities. Provisioning of effective care gets thwarted by equipment that is unavailable, non-functional and outdated and One fourth of the equipments in facilities were rendered non-functional biting the dust. 
As illustrated in Fig 5.2.2, for subcenters only one component had adjusted eigenvalue greater than one explaining 33.8\% variation. The principal component was represented predominantly by equipments enabling delivery such as delivery forceps, cord cutting scissors and sterilizers. On the other hand, for primary health centers illustrated in Fig 5.1.2, two components explaining 53.29\% variance explained the common variance. Principal component was characterized by neonatal care like availability of neonatal resuscitation mask, resuscitation kit, feeding tubes and radiant warmer for babies which is congruent to average availability scores. Further, delineating second component revealed the dominance of indicators associated with delivery preparedness. Marking a dissimilitude to component one, neonatal care factored negatively and strongly on second component.

In-depth conversations with health workers unfolded a nuanced account of obsolete methods of care or denial of care which they have to resort to due to unavailable/non-functional equipments. Some of the equipments were not germane to requirements and were supplied in surplus making it redundant e.g. few subcenters received around twenty thermometers each in the month preceding to survey indicating insufficient allocation of resources.

"We don't have equipment and infrastructure for delivery. We couldn't afford labour table with meagre funds and are conducting deliveries on wooden plank without sterilizer. Once a pregnant lady suffered the lacerations which needed suturing but I didn't have necessary kit, it endangered her life and finally succumbed to complications. I am reeling under guilt since then. What was my training worth?"

Health worker, Subcenter

\subsubsection{Infection Control Protocol}

Results indicated that healthcare settings lacked robust infection control infrastructure and no Healthcare Associated Infections (HAl) surveillance system existed in place. The list of tracer items incorporated practices like incineration of infectious materials, use of disinfectants and gloves, availability of deep burial pit and colour coded dustbins etc. For subcenters, more than one third facilities had only $25 \%$ items and 17 facilities were compliant to more than half the requirements. The readiness of NTPHC's were obsequious as compared to PHC's, the domain scores for facilities, on an average, were in range of $40 \%-90 \%$ for PHCs's and 0\%-80\% for NTPHCs. CHCs and DH however, were complying with $79 \%$ and $75 \%$ requirements respectively.

PCA results for subcenters conceded principal component explaining $41.22 \%$ variance and weighed positively by presence of sewerage system and appropriate disposal of waste material. Whereas, for primary health centers, proportion of variance explained by first two components with eigenvalues greater than one was $77.29 \%$. First component was characterized by the availability of guidelines and infection control protocol followed by segregation and disposal of waste material. Obversely, component 2 was positively associated with the presence of sewage system and negatively associated with adequate hypodermic syringe for single use. 
On an institutional level, healthcare facilities should work to foster organizational attributes and it should be endeavored to implement systems throughout organization that are prompt and reinforces the embodiment of infection control and prevention in all aspects of care. Even though health workers were conversant with standard operating procedures and guidelines, lack of supplies and infrastructural constraints made it difficult to practice standard precaution. No constant replenishing of exhausted materials such as gloves and needles was in place and health workers were purchasing it locally with the untied funds. All health workers griped about grappling with problem of waste disposal as they have to dig kutcha pit every time for disposing syringe/needles in the absence of pucca pit in the premises. Health workers also narrated the absence of government ownership of buildings as a stumbling block for adherence to infection control as observing hygiene/sanitation also hinges on owner of buildings from where they are running the facility as they reside cheek by jowl in same building.

"Ours is rented building and landlord has insisted on keeping his livestock and fodder in same building. There's gutter next to building and mosquito scourge due to that. Our woes doesn't stop here, since this building is situated on river bed; we can't bury the waste here, so we carry used up dressing material, syringes etc. $30 \mathrm{kms}$ away to our PHC for disposal."

MPW, Subcenter

\subsubsection{Diagnostics services}

Diagnostic capacity of facilities surveyed is represented in Fig 3. Subcenters are stipulated to provide very basic diagnostic tests such as Haemoglobin, Urine, Blood Sugar and Blood slide for malaria; nevertheless, it could effectuate only $43 \%$ readiness score. Less than one tenth facilities had all the four tracer items and more than one fourth of facilities were traipsing around $25 \%$ domain scores indicating suboptimal performance. PHCs, on an average, were also obscured by low readiness reaching just $50 \%$ score and scarcely any facility was conducting $2 \%$ sputum testing and possessing reagents and testing kits such as $\mathrm{KOH}$ solution, gram's iodine and safranin stain. Further, all NTPHCs except one was performing at less than $10 \%$ score and $63 \%$ facilities were unequipped with even basic kits like $\mathrm{Hbmeter}$, colorimeter and urine dipstick. There was serious vacuity connected with diagnostic capacity for Non communicable diseases and radiology in $\mathrm{CHC}$ and District hospital as none of them was providing services like memography, colposcopy, endoscopy, stress test, coomb's test, Pap smear and CT scan. On the sanguine side, all the hospitals were designated as Diagnostic Microscopy Centers conducting an array of serological, pathological and microbiological tests like urine analysis, stool analysis, RA factor test, VDRL test, LTF test, RPR for syphilis and ultrasound etc.

Two components following PCA were revealed to have eigenvalue greater than 1 explaining $82.52 \%$ common variance in subcenters. The principal component was highest loaded on availability of urine albumin and sugar testing kit and the component two was characterized by the collection of sputum samples in facility. Further, Horn's parallel analysis indicating first two components to be extracted explained $61 \%$ common variance in Primary health centers. Rotated factor loadings on principal 
component was denotative of presence of reagents like colorimeter, safranin stain followed by availability of functional microscope in laboratory. Whereas, second component evinced the presence of reagents and consumables required for testing of Tuberculosis with the loadings ranging from 0.19 to 0.61 .

In-depth interviews with laboratory technicians divulged that even if facility is having diagnostic capacity, other conditions are not conducive for operations. Also, geographical inaccessibility to the point of care induces a choice trade-off between public and private providers and the patients generally chose later due to time flexibility; thereby, contracting the demand for diagnostic services.

"I am unable to conduct the TB test due to dearth of water and space. Dental technician consults in the same room and his patients are always anxious of contracting infections. I have to fetch water manually from a hand pump situated $2.5 \mathrm{kms}$ away, sometimes it's not feasible to go, due to which I am always susceptible to infections. The equipments are getting corroded by rust and chemicals are expired; I haven't bothered to ask for new supplies as I know it would just sit idle.

Lab technician, $24 * 7$ Primary health center

\subsubsection{Medicines}

Provisioning of affordable, high quality and appropriate essential medicines is a quintessential component of a well-functioning health system. Fig 3 illustrates the distribution of health facilities based on availability of non-expired drugs. Essential medicine domain comprised a list of 38 drugs for subcenters and on an average, they were stocked with $52 \%$ drugs. Only half the facilities had anti-allergic and antibiotics, moreover, average availability of injectables and fluids was reprehensible with a score of $2 \%$. In a more expansive drug list and consumables; PHCs were stocked with $37 \%$ of medicines whereas NTPHCs egregiously had less than one fourth of medicines. No primary health center had insulin, inhalators and injectibles like amikacin, kanamycin and streptomycin, drugs for mental health disorders, antidotes for poisoning, expectorants, drugs for cardiovascular diseases and consumables like spinal disposable needle. On a sanguine note, all PHCs were stocked with vaccines for immunization and basic first aid kit. The domain score for $\mathrm{CHCs}$ and District hospital was $63 \%$ and $60 \%$ respectively, although, hospitals were also bereft of adequate consumables like mucus sucker, catheters and drugs required to treat non-communicable disease conditions.

The results of PCA for subcenters elucidated first four components with eigenvalue greater than 1 explaining 53.38\% common variance. Rotated component loadings for principal component underscored positive and heavy loadings on consumables for first aid like cotton bandages, povidone iodine solution followed by anti-allergens and antibiotics. Subsequent components were characterized by availability of antibiotics, zinc sulphate tablets and Vitamin syrup. Furthermore, for primary health centers, the variables possessing heaviest loadings on principal component were positively associated with injectibles, consumables viz intracath canulas, gloves and surgical spirit. In a dissimilitude to first component, 
injectibles however, weighed down the second component but were positively associated with drugs related to blood pressure and cardio-vascular diseases such as nifedipine, isosorbide and glyceryl trinitrate. First two components explained $32.18 \%$ common variance for primary care institutions.

Inadequate supply of drugs was identified as paramount domain leading to patient-provider wedge. The medicines received were not contingent to the needs as the morbidity profile of study area is dominated by blood pressure related and non-communicable diseases but the drugs addressing these conditions were absent in supply. Moreover, basic drugs for fever, gastrointestinal problems and antibiotics were reported to be exiguous.

"People come every-day and demand antibiotics and anti-allergens but we seldom receive it in stock. They hurl insults at us and proclaim why do you even bother to come to facility when you can't provide us with anything? They further insist upon administering steroids and sometimes we acquiesce otherwise they bad mouth us."

-Pharmacist, Sub center

At PHC level, the average time taken for medicine to reach post indenting was six weeks and the same was eight weeks for subcenters. Among the medicines which were unavailable in subcenters at time of survey, nearly $20 \%$ were out of stock for 3-6 months whereas, $40 \%$ were out of stock for more than 6 months. However, half of tracer drugs, surgical and suture items adapted from essential drug list were not procured and supplied ever by state medical supply corporation. Ensuring availability and optimizing expenditure is incumbent upon minimizing/eliminating wastage of drugs including pilferage, misuse and expiry. Expiry of medicines was identified as debilitating issue plaguing all the facilities, thereby, jeopardizing already constraint supply of medicines. Majority of health workers piqued over supply of drugs with very short shelf life. And it was more common for drugs related to vertical health programmes such as Vitamin A, IFA tablets, albendazole etc. The facilities didn't adhere to scientific inventory management method of First Expiry, First Out and 30\% facilities were found having stock of expired medicines.

"I received 1000 PCM tablets to last 13 months. Panting, exasperated an elderly female came to facility last week after trudging a distance of 2 hours down with fever but the medicine was out of stock and she got offended and dejected. Next day only Tikka express delivered boxes of expired ORS. Naturally, I expressed dissent, but they forced it upon me saying it's your buck to deal with."

Pharmacist, Sub center

\subsection{SERVICE SPECIFIC READINESS}

Fig 6. Presents a succinct snapshot of package specific composite measures capturing the capacity of facilities to deliver broad spectrum of services. Substantial heterogeneity in the readiness scores was found across the packages and facility types underscoring variation in the quality of care. On an average, 
the readiness of district and sub district hospitals across platforms were colossally different from facilities at the lower hierarchy of pyramid. Reproductive, maternal and child health were identified as better performing as facilities had an average of $91 \%, 70 \%$ and $66 \%$ of requisite components and supplies for provision of immunization, family planning and maternal and child care. The dissimilitude amongst district hospital and lower level health centers for birth preparedness and complication was colossal with district hospital effectuating $75 \%$ of desired inputs and processes for delivery care, whereas NTPHC's and sub centers were impaired with decrepit standards having $23 \%$ and $27 \%$ composite scores respectively. Adding a caveat, onlyone tenth of sub centers and NTPHC's reported providing obstetric and newborn services highlighting major lacunae in the delivery care. The readiness of services under Disease Control Program aiming to bridle the prevalence of vector borne diseases exhibited similar pattern with sub centers having disconcertingly low scores. These results reflect some compelling evidence on inadequate level of preventive care at the peripheral level which is otherwise indispensable for low resource settings due to cost effectiveness. In general, overall readiness for management of Tuberculosis and leprosy comprising screening, referral and follow up on cases with confirmed diagnosis and prescribed treatment was $60 \%$ for higher level health facilities which is quite suboptimal for the priority targeted interventions. The score was subjacent at $30 \%$ for sub centers and lowest at $15 \%$ for NTPHC's as compared to other levels of care. The capacity of facilities to optimally diagnose and treat noncommunicable diseases across the continuum of care is abysmally low across all levels of care while the population grapples with high burden of non-communicable diseases leading to gargantuan demandsupply gap wedge. For respiratory conditions, though $81 \%$ facilities provided services but no facility had all tracer commodities and staff for administering services and were equipped with $38 \%$ of recommended requirements only. Concomitantly, while secondary care hospitals provided $38 \%$ of services for diabetes, these figures nosedived for primary care facilities and further deflated down to $0 \%$ for sub centers connoting none of the sub center had any diabetes related services and preparedness. However, the degree of readiness for cardiovascular diseases oscillated between with $29 \%$ for hospitals to merely $6 \%$ for sub centers and was mostly characterized by drugs for treatment of blood pressure. Although lower level facilities are relegated with basic functions like screening, referral and monitoring of symptoms of cancer, they failed to comply with the norms and were completely defunct in provision of services. The provisioning of mental health services was also appalling with service provision being null and void in hospitals and only counselling related services provided in primary care. Only $6 \%$ of public health workers in rural peripheries reported providing counselling services albeit they were not trained in counselling, thereby, casting a shadow over the quality and effectiveness of such services. This is particularly alarming in a tense geopolitical scenario such as for people living along the borders are for most part powerless, with little control over their environment and are also extremely vulnerable to physical and psychological injury. It is also tenable to have robust emergency preparedness and response as people residing in study sites find themselves in direct line of fire as the area is strewn with landmines and is perturbed by cross border shelling and militarized encounters. Emergency referral is critical to improving outcomes in such time sensitive conditions. In spite of this district having high exposure to risk, emergency preparedness scores were low at merely 30\% for district hospital and $9 \%$ for sub centers with no priority preparedness being for facilities at the precipice of border and less venerable to risk facilities. 
Poor readiness was found for indicators like presence of bunkers in vulnerable facilities, availability of bullet proof ambulances, strong communication network and availability of surgeons and blood transfusion etc.

\subsection{Creating Vulnerability Index}

Vulnerability index is a parsimonious yet practical tool to adjust healthcare delivery based on access and gauge the relative exigencies faced by the various facilities in the context of difficult/fragile setting. In our area context; facilities are navigating multitude of structural, non -structural and functional challenges such as topographical, seismological, security, infrastructural and presence of vulnerable communities and groups. The index was constructed incorporating myriad of indicators adapted and modified from High Level Expert Group Report on Universal Health Coverage for India (Planning Commission of India, 2011) and the scores for each indicator were assigned across facilities based on exposure, sensitivity and resilience after having deliberations with district administrative authorities. Operational vulnerability of facility was chosen as the focal point based on the axis of external and internal conditionals i.e. vulnerabilities spanning susceptibility and exposition to shocks and stresses as external conditionals and incapacities/capacities of facilities to amplify/attenuate the stresses and shocks as internal conditionals(Birkman and Wisner,2006). The degree to which a facility delivers effective care is sensitive to the stressors emanating from internal or external environment. The method coalesced 13 indicators representing vulnerability on a scale of 0 to 50 . The index takes into account variables capturing a) isolation factor such as travel time to healthcare facility by walk/other modes of transport, difficulty of terrain, availability of transport, distance to major district roads and public transport b) density of health workers given population density and geography c) infrastructural impediments such as condition of the roads and availability of government accommodation d) security threat such as cross border firing zones and militancy affected areas and e) marginalized population such as proportion of tribal population in the catchment area. The detailed description of indicators and weighting strategy for vulnerability index calculator is propounded in Table 1, Appendix.

Following narrative echoes the various layers of vulnerabilities experienced by providers in our context.

"As you can see that our facility is after crossing border's fencing in zero line and firing/shelling ensues erratically at drop of the hat. Every day we have to cross Army enclosure post cordoning formalities encumbered with fear and uncertainty. No public transport comes here, I have to come in my bike till the fence which costs me ten thousand rupees every month out of my fourteen thousand rupees salary. Moreover, after crossing the fence, we have to walk for $2 \mathrm{kms}$ amidst thick forests and bushels. During winters, when fog gathers; it exacerbates risks as leopards prowl here. During the days when shelling catapults, we have to take cover and use alternate routes via gullies to reach facility. Last month only, a bullet ricocheted off next to me and I panicked hard".

-Male MPW(Contractual, Medical Aid Center)

Page $18 / 31$ 


\subsection{Determinants of Health facility readiness scores}

Health facility readiness score was modelled as a function of covariates and table 2 presents the results of generalized ordered modelling where a unit increase in independent variable, alters the probability of falling in the jth alternative by marginal effect in percentage terms. For each facility, general readiness score was calculated by coalescing scores across five dimensions encompassing a legion of tracer indicators. The distribution of average facility scores was fitted into quartiles to create a three-level ordinal scale for health facility readiness. The dependent variable is polychotomous and outcome falls into three quartile categories i.e. high, medium and low. Vulnerability score, facility type and administrative area reflected the largest magnitude of marginal impact on outcome probabilities. Medium vulnerability score was found to be associated with poor readiness as it was $14.7 \%$ more probable than lower vulnerability to result in poor readiness. However, facilities with menacing vulnerability scores were $19.1 \%$ more likely to have poor outcome and conversely, $19.8 \%$ less likely to yield good readiness scores compared to the reference category. It can be surmised from table that frequent supervision and monitoring is associated with $9.2 \%$ lesser probability than sporadic supervisory visits to beget poor readiness. Although better supervision was more likely to have good readiness, this finding was statistically insignificant. Facility type was found to be another impregnable factor impacting the dependent variable as physician led clinics and hospitals were $24.7 \%$ less and $14.3 \%$ more likely than peripheral sub centers to have poor and good readiness respectively and in terms of magnitude, the effect of facility type was more extreme at lower levels of readiness score. Similarly, the impact of administrative block was quite cogent in explaining the readiness scores as facilities in health block Mendhar had $14.4 \%$ lesser and $14.7 \%$ more probability to culminate poor readiness vis a vis block Mandi. Understaffing of facilities however, was significantly more likely than no vacancy of core health worker to have poor readiness by $10.3 \%$.

\section{Table 2: Marginal Effects of the Covariates for each outcome using partial proportional odds model}

\section{Discussion And Conclusion}

The study intended to augment the understanding of various components of supply side readiness and its associated factors. Our analysis of structural readiness revealed copious and pervasive gaps in basic capacity to deliver healthcare services. None of the facilities surveyed possessed all the resources required for providing services. The dissimilitude in readiness scores between various levels of facilities was confounding. Subcenters and new type primary health centers were particularly low performing and invariantly had less readiness Vis a Vis hospitals. Same findings reverberates through other studies across different regions in LMICs where hospitals were found to report significantly greater input availability and concentration of resources than health centers and dispensaries(Bintabara et al, 2019; Ssempiira et al; 2018; Winter et al; 2017; lyer et al, 2015; Boyer et al,2015). The challenges confronting peripheral rural institutions are vexed ranging from workforce shortage, poor monitoring and evaluation, suboptimal budgetary allocation and lack of community participation. Non availability of basic amenities 
like water, electricity and basic medicines has profoundly undermined the functioning of facilities. The facilities lacked the presence of all resources simultaneously for effective delivery; for example if SBA trained workers were available at the facility, the labour room was absent; rendering the skilled workforce redundant to provide obstetric care. As a corollary, poor and vulnerable individuals relying on free services in peripheral facilities remains underserved underpinning inverse care law that conjectures that availability of good medical care tends to vary inversely with need of population served. Spatial concentration of resources intertwined with rural-urban dichotomy entailed that there was hardly referral system operating in public health set-up of district.

Our study revealed abysmal score for basic infrastructural amenities and diagnostic capacity with the lowest readiness for NTPHCs. The NTPHCs exhibited staggering performance for other domains as well with overall general readiness score of $35 \%$. The NTPHCs were upgraded from Allopathic Dispensaries and few subcenters to streamline and strengthen existing health system, yet, half of the NTPHCs were operating from unsafe rented buildings and none of them had medical officer or functional laboratory. People residing in catchment area of these facilities are compelled to travel district/block headquarters for diagnostic services and purchase medicines from open markets at exorbitant rates. These results concur with Shawon et al (2018) who reported that in Bangladesh, facilities at all levels had lowest scores for diagnostic capacity. Another study conducted in India reported absence of diagnostics for routine blood, urine and stool examination especially in Empowered Action Group states such as Assam where only $20 \%$ primary health centers had basic laboratory facilities (Ninama et. al, 2014)

The unavailability of basic drugs contravenes the ethos of UHC as expenditure on medicines is dominant cause of catastrophe and impoverishment at household level. The OOP on drugs for ambulatory care is $59.73 \%$ of total OOP in rural areas of Jammu and Kashmir (Calculated from NSSO, $71^{\text {st }}$ round). Our estimates of $31 \%$ average availability of medicines in PHCs bears similitude with the study conducted on pediatric drugs in PHC in a district of Kashmir with $34.8 \%$ availability and 115 days of stock-out (lqbal et al, 2015). Recently, Jammu and Kashmir government adopted Free Drug Policy in 2016(J\&K Government Order No. 142-HME, Annexure 1.2016) entitling citizens to free essential generic drugs in order to mitigate burden of healthcare costs via Jammu and Kashmir Medical Supply Corporation(JKMSCL). However, multiplicity of supply chain issues like incongruity between procurement and requisition, shortage of corpus funds and payment delays to suppliers, delay in lifting medicines from regional drug warehouse, convoluting tender process involving multiple corrigendum and procurement of consumables with high commission were impeding drug provisioning via JKMSCL.

Our findings accentuated lowest readiness for Non-Communicable diseases as compared to the targeted packages like Immunization, Maternal and Child services, Family Planning etc. This is consonant with results reported from similar studies. Studies conducted in constraint settings divulged that facilities demonstrated lower capacity for treating NCDs as compared to infectious diseases (IHME, 2014; Jigjidsuren et al; 2018; Mutale et al, 2018). Despite NCD being biggest contributor of disease burden; it has been put on the backburner and integration on NCD interventions into framework of public health delivery has been quiescent. Specifically, the acknowledgement of epidemiological transition by local 
health system and infirmities in the surveillance and integration of chronic diseases in health information system has led to lack of reliable data for evidence based policymaking.

In order to unpack the contextual factors, our study examined the determinants of health facility readiness and found greater level of vulnerability, sporadic monitoring visits and lower level of facility to have higher probability of having poor readiness which was symptomatic with results from other studies in low resource and difficult settings(Bintambara et al, 2017;0yekale et al, 2017). Absence of monitoring has been associated with preponderance of workforce absenteeism and defunct rural facilities. Also, the role of supply side vulnerabilities which is a complex dimension works via different pathways to impede the service delivery. The triple whammy of geographical remoteness, disturbed security scenario and lack of basic amenities results in cannibalization of health workforce via internal adjustment mechanism. Further, armed conflicts, internal disturbances and other types of unrest create a generalized state of insecurity that often makes maintaining a minimally functional health system really impossible (Nickerson, 2015). Though local administration recently started distribution and training of conflict zone first aid kits in firing Zone $A$ (most disturbed) of border, but lack of resources to purchase bullet proof ambulances, absence of underground bunkers in facilities and exposure of health workers to the vagaries of shelling continues to deprive border population of even rudimentary health services.

\section{Policy Recommendations}

We discerned from our study that it is pertinent to bolster existing primary care facilities in this setting rather than opening new ones. There is need to circumspect about the issue of staff shortage and absenteeism which is particularly nettlesome as staff engages in dereliction of duty with impunity. Change in organizational behavior using surveillance; characterized by practice of record keeping, frequent monitoring of employee absence behavior, detection of absences and setting of thresholds for individual action which if breached set off a cascade of management action oriented measures are found to be some strategies effective in LMICs(Kiwanuka et al,2011). Further, introduction of biometric monitoring system can be explored to reduce absenteeism e.g. a pilot project in Karnataka, India providing real time tracking of attendance in PHCs ameliorated absenteeism amongst paramedical staff. Intervention analogous to Chhattisgarh Rural Medical Corp (CRMC), an integrated incentive scheme to attract and retain doctors in underserved, remote and difficult areas including conflict areas encompassing financial incentives, residential accommodation, life insurance and extra credits for postgraduate level to eligible doctors can be envisioned in the study area as CRMC reduced vacancies of doctors from $90 \%$ to $45 \%$. Concomitantly, action oriented methods like community based monitoring, social audits, Jan Sunwayis coupled with incentives based on vulnerability index (higher payments for hard to reach locations) should be considered. There should also be copious amount of budgetary allocation either by creating fiscal space or attracting aid to purchase armored ambulances and fortifying facilities against bullet/shelling attacks in zone A (most disturbed) of border areas.

Another formidable barrier of geographical inaccessibility can be transcended by setting up telemedicine nodes in inaccessible facilities. Hybrid model of telemedicine using both store and forward 
(asynchronous) as well as real time (synchronous) connectivity within Hub and Spoke framework can be introduced to provide accessible, affordable and quality healthcare. Subcenters in remote catchment areas acting as spokes can be transformed to e-subcenter where telemedicine nodes can be established connecting them via satellite link with hub acting as specialty nodes. There is cogent evidence of benefits by introducing such models in collaboration with India Space Research Organization (ISRO) in tribal, hilly and remote areas of India. ISRO in collaboration with State governments in India covered 384 hospitals with 60 specialty hospitals connected to 306 remote/rural/district/medical college hospitals and 18 mobile telemedicine units. An intervention congruous to this model can be explored in our contextual setting.

The conflation of quantitative and qualitative insights highlighted the need to rejuvenate peripheral health facilities specifically to provide obstetric and new born care, NCD care and emergency preparedness. The efficacy of these facilities are particularly tethered by lack of diagnostics and essential medicines. Targeted interventions entrenched in Central Government's flagship schemes like setting up government pharmacies dispensing generic drugs at affordable prices and pharmacies specially established to provide drugs, implants, surgical disposables and other consumables for cancer and heart disease at heavily discounted rates should be prioritized to fill this gap and complement drug supply via corporation. Further, under aegis of Ministry of Health and Family Welfare, Indian Council of Medical Research has drafted first National Essential Diagnostics List (NEDL) to be implemented soon appreciating the need to improve availability of quality diagnostics. Implementation of NEDL should be prioritized in the study area and integrated with telemedicine to improve patient outcomes and reduce out of pocket expenditure. It is also suggested to implement WHOs package of essential NCD disease interventions for primary healthcare in low resource settings (WHO PEN) providing evidence based clinical guidelines to improve access and quality of NCD services delivered at primary health-care facilities.

\section{Declarations}

\section{Ethics approval and consent to participate}

The protocol for this study has been approved by ethical committee of Indian Institute of Technology Madras. All human subjects in the study were asked for their consent, and had provided their consent before data collection. Both oral and written consent was obtained by facility incharges and official permissions were obtained from Directorate of Health and Family Welfare, Jammu and Kashmir and district health authorities to conduct facility survey.

\section{Consent for publication}

Not applicable

\section{Availability of data and materials}


Data collection is done through primary survey and is available upon request.

\section{Competing interests}

The authors declare that they have no competing interests.

\section{Funding}

There is no external funding involved in the project.

\section{Author's contributions}

VRV is involved in conceptualizing the research and its methodology, conducted the field work, analysis and led the writing of manuscript. UD was involved in methodological design and assisted in revising original manuscript. Co-author has read and approved the original manuscript.

\section{Acknowledgements}

We would like to thank state health and district health authorities for methodological inputs and providing logistic support. We also extend our sincere gratitude to Anganwadi workers in the district for providing logistic support during fieldwork particularly in hard to reach and inaccessible areas.

\section{References}

Birkmann J, Wisner B. Measuring the unmeasurable: the challenge of vulnerability. UNU-EHS; 2006.

Bintabara D, Ernest A, Mpondo B. Health facility service availability and readiness to provide basic emergency obstetric and newborn care in a low-resource setting: evidence from a Tanzania National Survey. BMJ open. 2019 Feb 1;9(2):e020608.

Bintabara D, Nakamura K, Seino K. Determinants of facility readiness for integration of family planning with HIV testing and counseling services: evidence from the Tanzania service provision assessment survey, 2014-2015. BMC health services research. 2017 Dec;17(1):844.

Biswas T, Haider MM, Gupta RD, Uddin J. Assessing the readiness of health facilities for diabetes and cardiovascular services in Bangladesh: a cross-sectional survey. BMJ open. 2018 Oct 1;8(10):e022817

Boerma T, Eozenou P, Evans D, Evans T, Kieny MP, Wagstaff A. Monitoring progress towards universal health coverage at country and global levels. PLoS medicine. 2014 Sep 22;11(9):e1001731. 
Boyer C, Jackson E, Bawah A, Schmitt M, Awoonor-Williams J, Phillips J. Estimating indices of health system readiness: an example from rural northern Ghana. The Lancet Global Health. 2015 Mar 1;3:S14.

Donabedian A. The quality of care: how can it be assessed?. Jama. 1988 Sep 23;260(12):1743-8.

Glorfeld LW. An improvement on Horn's parallel analysis methodology for selecting the correct number of factors to retain. Educational and psychological measurement. 1995 Jun;55(3):377-93.

Greene JC, Caracelli VJ, Graham WF. Toward a conceptual framework for mixed-method evaluation designs. Educational evaluation and policy analysis. 1989 Sep;11(3):255-74.

Horn JL. A rationale and test for the number of factors in factor analysis. Psychometrika. 1965 Jun 1;30(2):179-85.

Institute for Health Metrics and Evaluation (IHME). Health Service Provision in Kenya: Assessing Facility Capacity, Costs of Care, and Patient Perspectives. Seattle, WA: IHME, 2014

Iqbal MJ, Geer MI, Dar PA. Availability and Stock Outs of Paediatric Essential Medicines Across Different Levels of Care in One District. Indian Journal of Pharmacy Practice. 2015 Oct; 8(4): 171.

Islam F, Rahman A, Halim A, Eriksson C, Rahman F, Dalal K. A" Model Quality Improvement System" for Maternal and Newborn Health Services Applicable for District and Sub-district Level Hospitals in Bangladesh: Description of Model Development Process. Health Syst Policy Res. 2016;3:1.

lyer HS, Kamanzi E, Mugunga JC, Finnegan K, Uwingabiye A, Shyaka E, Niyonzima S, Hirschhorn LR, Drobac PC. Improving district facility readiness: a 12-month evaluation of a data-driven health systems strengthening intervention in rural Rwanda. Global health action. 2015 Dec 1;8(1):28365.

Jigjidsuren A, Byambaa T, Altangerel E, Batbaatar S, Saw YM, Kariya T, Yamamoto E, Hamajima N. Free and universal access to primary healthcare in Mongolia: the service availability and readiness assessment. BMC health services research. 2019 Dec;19(1):129.

Kiwanuka SN, Nalwadda C, Pariyo GW, Ssengooba F, Rutebemberwa E. Interventions for managing absenteeism among health workers. Cochrane Database of Systematic Reviews. 2014 Sep 23;2014(9):CD009250.

.Kolenikov S. polychoric, by any other'namelist'. In2016 Stata Conference 2016 Aug 10 (No. 15). Stata Users Group.

Macarayan EK, Gage AD, Doubova SV, Guanais F, Lemango ET, Ndiaye Y, Waiswa P, Kruk ME. Assessment of quality of primary care with facility surveys: a descriptive analysis in ten low-income and middleincome countries. The Lancet Global Health. 2018 Nov 1;6(11):e1176-85. 
Maca K, Fernandes Q, Kanté AM, Bawah A, Condo J, Mutale W. Measuring health systems strength and its impact: experiences from the African health initiative. BMC health services research. 2017 Dec;17(3):827.

Ministry of Health and Family Welfare, Government of India. Report of the Task Force on Comprehensive Primary Health Care Rollout.

Mutale W, Bosomprah S, Shankalala P, Mweemba O, Chilengi R, Kapambwe S, Chishimba C, Mukanu M, Chibutu D, Heimburger D. Assessing capacity and readiness to manage NCDs in primary care setting: Gaps and opportunities based on adapted WHO PEN tool in Zambia. PloS one. 2018 Aug 23;13(8):e0200994.

Nickerson JW. Ensuring the security of health care in conflict settings: an urgent global health concern. CMAJ. 2015 Aug 11;187(11):E347-8.

Ninama R, Thakor N, Dund J, Kadri AM. Quality assessment of facilities available at primary health care centers in Rajkot district: a cross sectional study. International Journal of Medical Science and Public Health. 2014 Dec 1;3(12):1449-53.

Oyekale AS. Assessment of primary health care facilities' service readiness in Nigeria. BMC health services research. $2017 \mathrm{Dec} ; 17(1): 172$.

Planning Commission. High level expert group report on universal health coverage for India. 2011 Dec.

Shawon MS, Adhikary G, Ali MW, Shamsuzzaman M, Ahmed S, Alam N, Shackelford KA, Woldeab A, Lim SS, Levine A, Gakidou E. General service and child immunization-specific readiness assessment of healthcare facilities in two selected divisions in Bangladesh. BMC health services research. 2018 Dec;18(1):39.

Ssempiira J, Kasirye I, Kissa J, Nambuusi B, Mukooyo E, Opigo J, Makumbi F, Kasasa S, Vounatsou P. Measuring health facility readiness and its effects on severe malaria outcomes in Uganda. Scientific reports. 2018 Dec 18;8(1):17928.

Truth AU. No Health without a workforce. World Health Organisation (WHO) Report. 2013 Nov.

Wanzala M. Assessing Capacity and Performance of Health Systems Using Principal Component Analysis: Results from Cross Sectional Survey in Kakamega County, Western Kenya.

Winter R, Yourkavitch J, Wang W, Mallick L. Assessment of health facility capacity to provide newborn care in Bangladesh, Haiti, Malawi, Senegal, and Tanzania. Journal of global health. 2017 Dec; 7(2).

World Health Organization. Service availability and readiness assessment (SARA): an annual monitoring system for service delivery: reference manual. World Health Organization; 2013. 


\section{Table}

Table 2: Marginal Effects of the Covariates for each outcome using partial proportional odds model

Marginal Effects of the Covariates for each outcomes using Partial Proportional Odds

\begin{tabular}{|c|c|c|c|}
\hline Variables & $\begin{array}{l}\text { Outcome: Poor } \\
\text { Readiness }\end{array}$ & $\begin{array}{l}\text { Outcome: Fair } \\
\text { Readiness }\end{array}$ & $\begin{array}{l}\text { Outcome: Good } \\
\text { Readiness }\end{array}$ \\
\hline \multicolumn{4}{|l|}{ ability(Ref: Low) } \\
\hline \multirow[t]{2}{*}{$\bar{m}$} & $.147^{\star \star *}$ & $-.091^{\star \star}$ & -.135 *ᄎ* \\
\hline & $.191^{\star \star \star}$ & 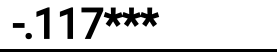 & -.198 *** \\
\hline \multicolumn{4}{|c|}{ ision(Ref: Infrequent) } \\
\hline !nt & $-.092^{\star \star}$ & .007 & .0101 \\
\hline \multicolumn{4}{|c|}{ f facility(Ref: Sub } \\
\hline$\overline{\mathrm{HC} / \mathrm{DH}}$ & $-.247 * \star \star$ & $.189 * *$ & $.143^{\star \star \star}$ \\
\hline \multicolumn{4}{|l|}{ Ref:Mandi) } \\
\hline cote & $-.076 *$ & $-.053^{\star}$ & $.108 * \star$ \\
\hline $\operatorname{ar}$ & $-.144 * \star$ & $.114^{\star \star \star}$ & $.147^{\star \star \star}$ \\
\hline \multicolumn{4}{|l|}{$\begin{array}{l}\text { Audits(Ref: } \\
\text { lent) }\end{array}$} \\
\hline !nt & -.004 & .005 & .042 \\
\hline \multicolumn{4}{|l|}{$\begin{array}{l}\text { osition (Ref: No } \\
\text { cies) }\end{array}$} \\
\hline cies & $-.103^{\star}$ & -.010 & -.021 \\
\hline
\end{tabular}

Figures 

Survey

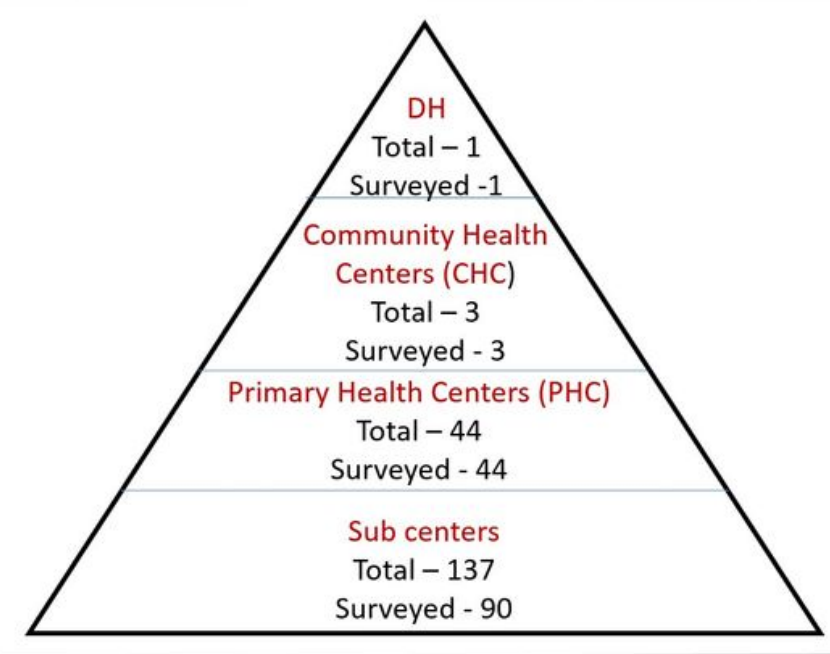

Closed Ended Questionnaire

Checklist:- Inrastructure, Amenities, Staff \& Guidelines, Equipment, Diagnostics and Infection Control Protocols

Open Ended Questionnaires

\section{Figure 1}

\section{Study Framework}

\section{SERVICE AVAILABILITY SCORES}

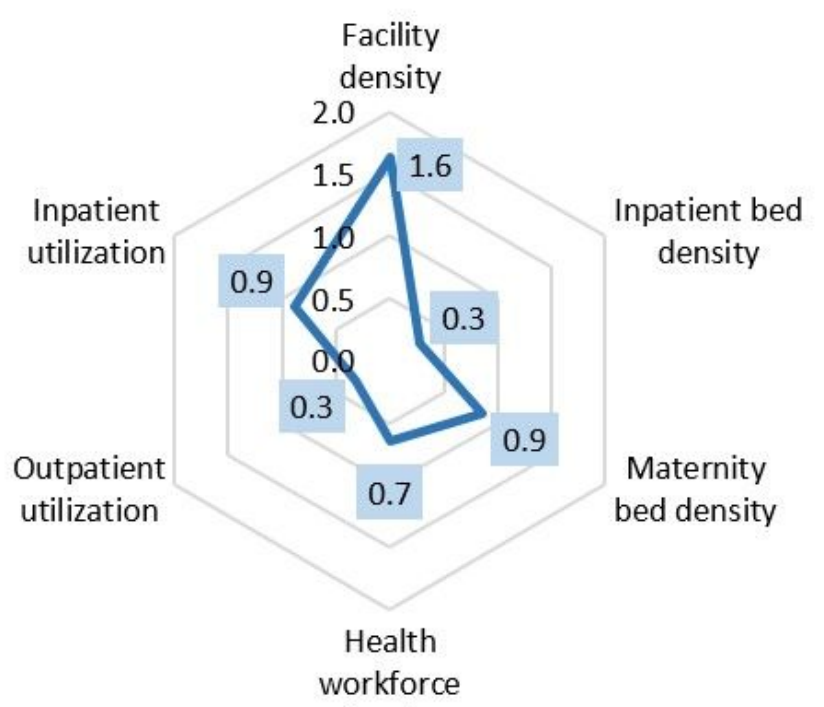

GENERAL READINESS SCORES

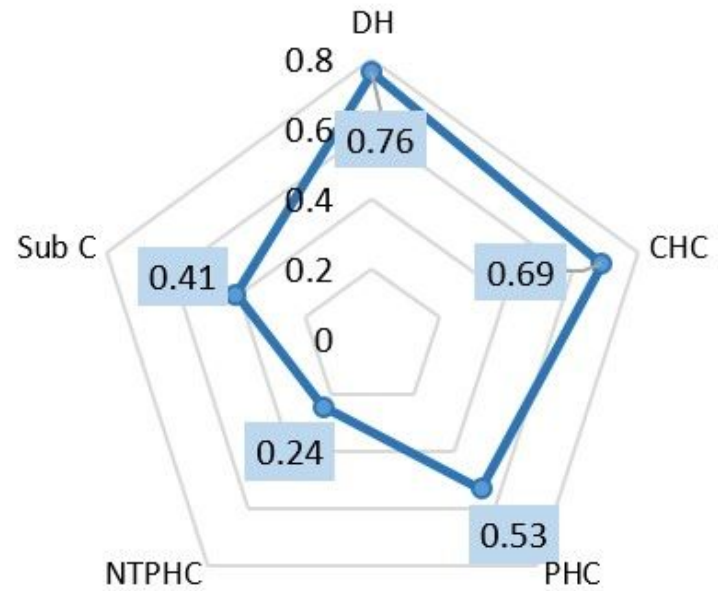

\section{Figure 2}

\subsection{Service Availability Scores. 2.2. General Readiness Scores}



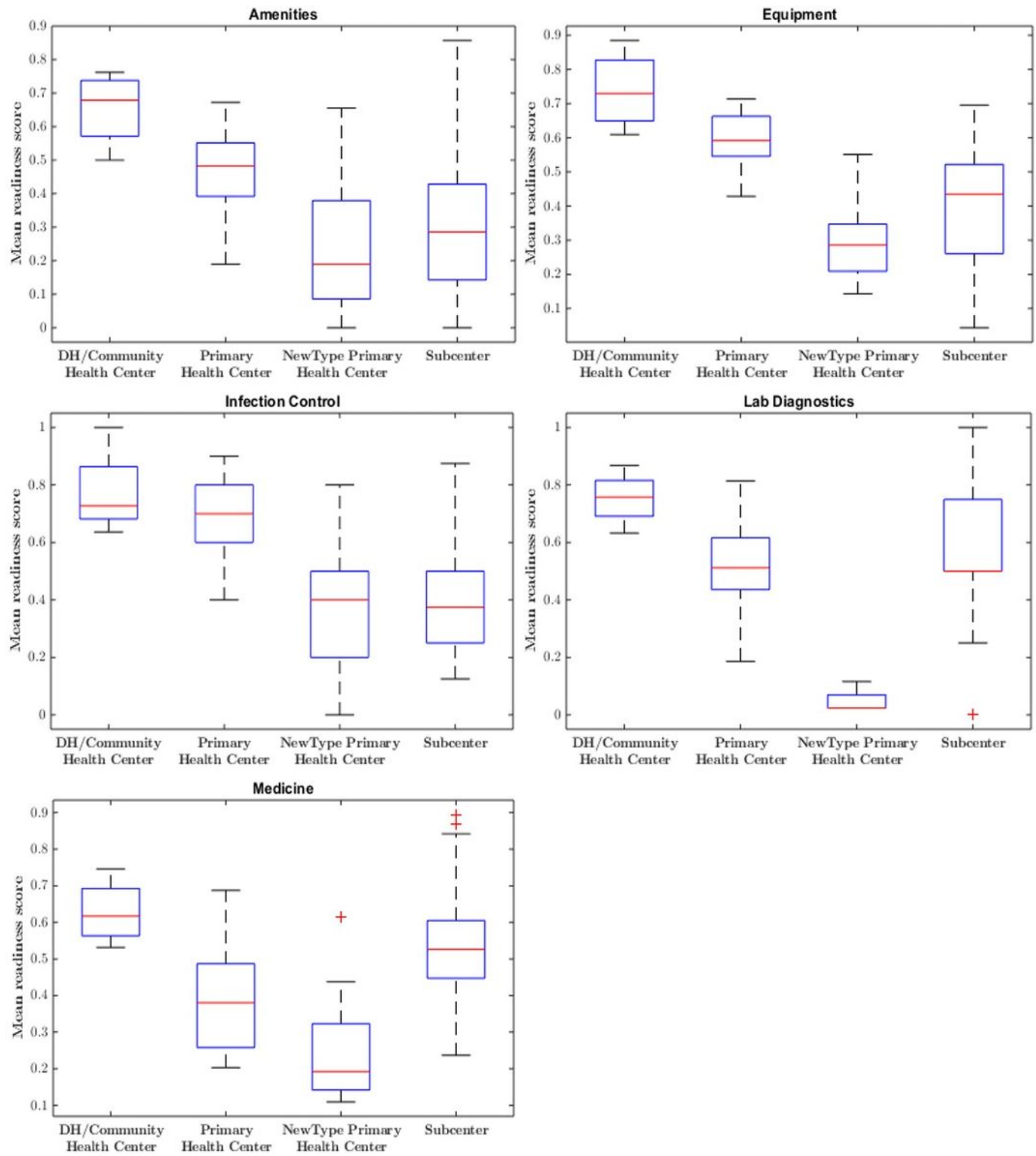

Figure 3

General Readiness Scores across Domains 


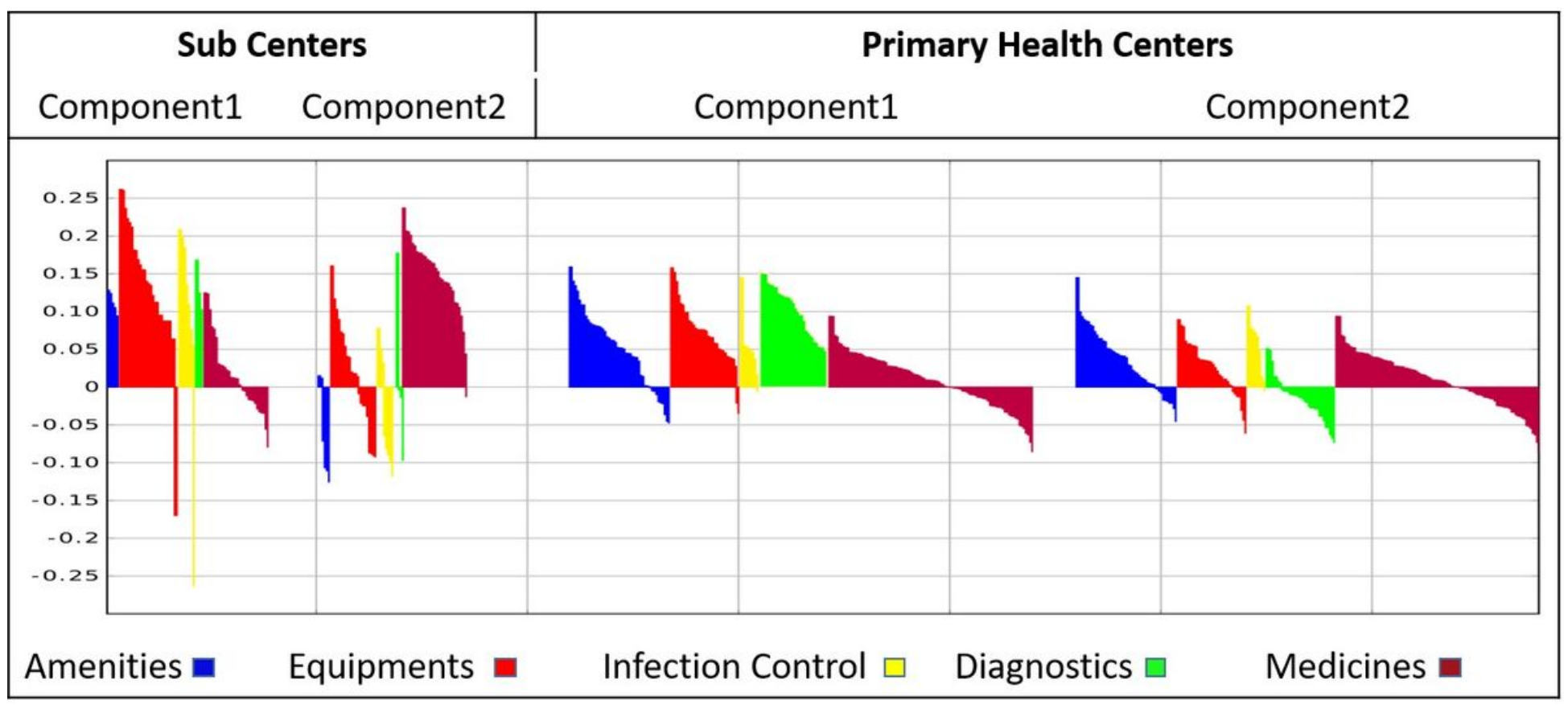

Figure 4

PCA loadings and variable rank order: General Readiness for Subcenters and Primary (incl New type) Health Centers 

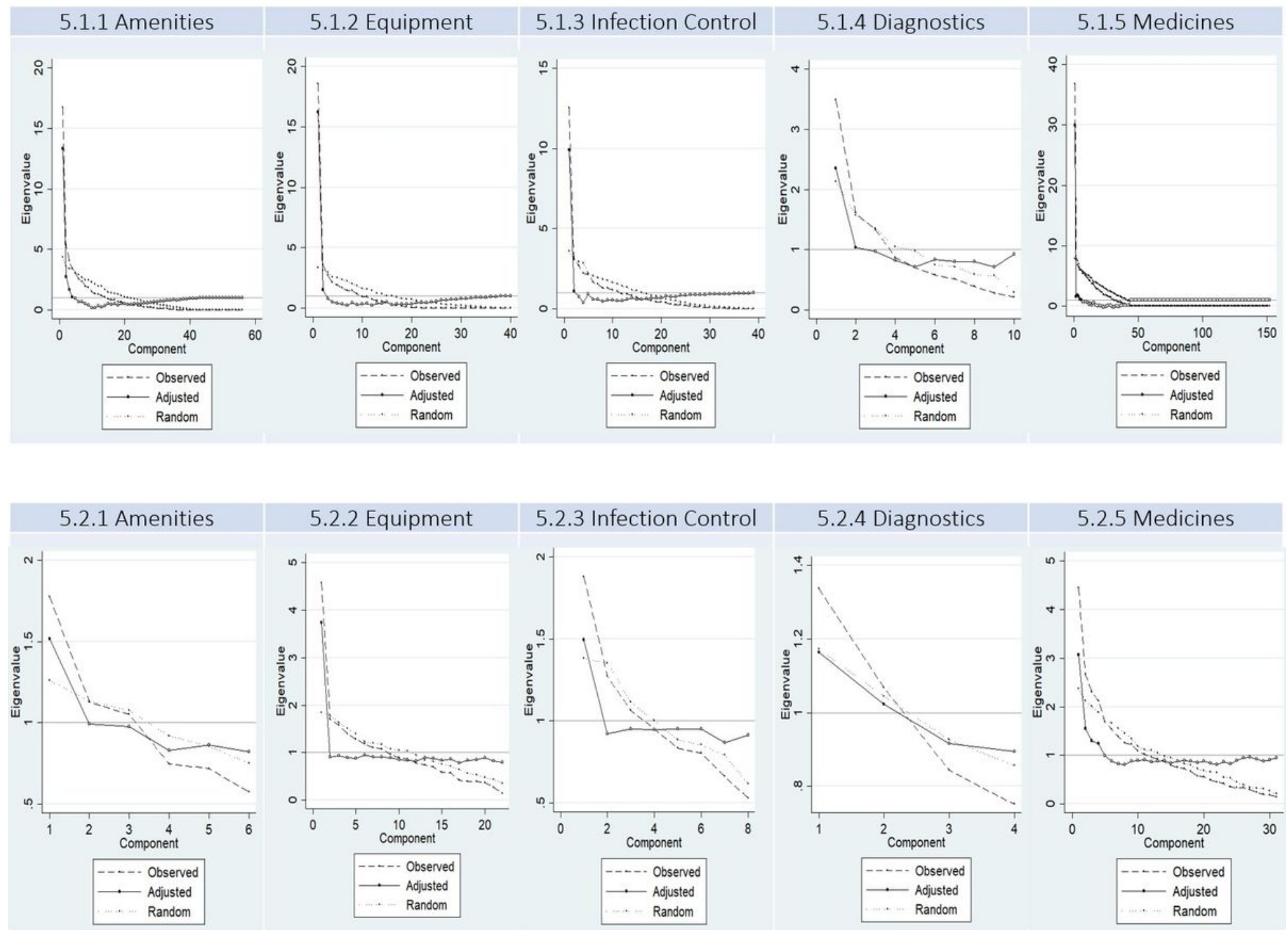

5.2.5 Medicines

\section{Figure 5}

5.1 Scree Plots: Primary Health Centers (Including Newtype Primary Centers). 5.2. Scree Plots: Health Sub-Centers 


\begin{tabular}{|c|c|c|c|c|c|}
\hline & $\begin{array}{l}\text { District } \\
\text { Hospital }\end{array}$ & $\begin{array}{c}\text { Community } \\
\text { Health } \\
\text { Center }\end{array}$ & $\begin{array}{l}\text { Primary } \\
\text { Health } \\
\text { Center }\end{array}$ & $\begin{array}{c}\text { New Type } \\
\text { Primary } \\
\text { Health } \\
\text { Center }\end{array}$ & Sub-Center \\
\hline Routine Immunization & 100 & 97 & 91 & 67 & 78 \\
\hline Family planning and contraception & 100 & 90 & 62 & 57 & 60 \\
\hline Basic surgical care & 89 & 64 & 51 & 20 & 22 \\
\hline School health services and adoloscent health & 88 & 88 & 67 & 51 & 45 \\
\hline Malaria and febrile diseases & 86 & 69 & 67 & 58 & 73 \\
\hline Maternal and child services & 84 & 75 & 70 & 50 & 53 \\
\hline General and Oral health & 81 & 65 & 48 & 30 & 28 \\
\hline Obstetric and Newborn Care & 75 & 66 & 47 & 23 & 27 \\
\hline Outreach services & 72 & 76 & 81 & 85 & 90 \\
\hline Disease control programmes & 69 & 52 & 50 & 46 & 40 \\
\hline HIV/STI diagnose and management & 69 & 40 & 34 & 18 & 29 \\
\hline Tuberculosis and Leprosy services & 68 & 46 & 38 & 15 & 30 \\
\hline Respiratory/chest diseases & 59 & 42 & 29 & 21 & 29 \\
\hline Diabetes & 42 & 33 & 19 & 11 & 0 \\
\hline Cardiovascular diseases & 29 & 17 & 15 & 10 & 6 \\
\hline Emergency preparedness & 30 & 22 & 14 & 11 & 9 \\
\hline Cancer & 9 & 0 & $\mathbf{0}$ & $\mathbf{0}$ & $\mathbf{0}$ \\
\hline Mental health & 0 & 0 & $\mathbf{0}$ & 8 & 6 \\
\hline Neurology & $\mathbf{0}$ & 0 & $\mathbf{0}$ & 0 & $\mathbf{0}$ \\
\hline
\end{tabular}

Figure 6

Availability of services in facilities, by platform

\section{Supplementary Files}

This is a list of supplementary files associated with this preprint. Click to download.

- appendix.docx

- Formulas.docx 